\title{
Case Series of Pneumomediastinum in COVID-19 Infection
}

\author{
Fakhreddine $\mathbf{S}^{1}$, J aber $\mathbf{R}^{2}$, Skaff $\mathbf{E}^{2}$, Salloum $\mathbf{S}^{3 *}$ \\ and Maatouk $\mathrm{A}^{4}$ \\ ${ }^{1}$ Infectious Disease Department, Saint George Hospital, \\ Hadath, Lebanon \\ ${ }^{2}$ Medical Student, Lebanese University, Lebanon \\ ${ }^{3}$ Department of Internal Medicine, Lebanene University, \\ Lebanon \\ ${ }^{4}$ Department of Pulmonary and Critical Care Medicine, \\ Covid 19 Program Director, Saint George Hospital, \\ Hadath, Lebanon \\ *Corresponding author: Salloum S, Department of \\ Internal Medicine, Lebanese University, PGYIII, Lebanon
}

Received: March 22, 2021; Accepted: April 09, 2021; Published: April 16, 2021

\begin{abstract}
Introduction: Pneumomediastinum is rare in viral infection of the lung however in COVID-19 patients it is more common.

Study Design: Case series of 14 moderate to severe COVID cases complicated by Pneumomediastinum admitted to Saint George Hospital over 4 months. Data was collected retrospectively from medical charts of the patients.

Results: Most of the patients were males. Average hospital stay was 15.21 days. Five patients $(35.72 \%)$ developed pneumomediastinum without any kind of mechanical ventilation during hospitalization. Around $35.72 \%$ of the patients were discharged and the average time till death was 8.8 days.
\end{abstract}

Conclusion: Pneumomediastinum can develop without any positive pressure ventilation in COVID-19 infection.

Keywords: Pneumomediastinum; COVID-19; Acute respiratory distress syndrome

\section{Introduction}

Spontaneous pneumomediastinum is rare in viral pneumonia [1]. Known risk factors for non-traumatic pneumomediastinum are: decreased lung compliance, age, the presence of an underlying lung disease such as interstitial lung disease or Chronic Obstructive Pulmonary Disease (COPD) and certain lung infections such as pneumocystis jirovecii pneumonia [2].

The exact mechanism causing a pneumomediastinum is unknown yet; however, the increase in alveolar pressure and diffuse alveolar injury seen in severe COVID-19 lung infections make the alveoli susceptible to rupture, especially in the setting of a pronounced cough [1]. In contrast, the occurrence of pneumomediastinum in mechanically ventilated patients can be multifactorial [3]. With the current approach of protective ventilation in patients with Acute Respiratory Distress Syndrome (ARDS), a decrease in the occurrence of the main signs of barotrauma (pneumothorax, pneumomediastinum and subcutaneous emphysema) was seen [4] Pneumomediastinum in COVID-19 ARDS is not associated with the classic barotrauma mechanism which refers to high transpulmonary pressure [5]. In fact, during the COVID-19 pandemic there was an increase in the incidence of pneumomediastinum and subcutaneous emphysema despite using the same protective lung strategy during mechanical ventilation [2]

The aim of this study is to describe a case series of patients with COVID-19 infection with pneumomediastinum and discuss the presence of common laboratory or clinical features among them.

\section{Study Design}

A retrospective study was conducted. Data was collected from the medical files of patients admitted to Saint George hospital in Hadath, Lebanon, from September 1, 2020 to December 31, 2020.
All the cases with pneumomediastinum were selected. A clinical staging of the patients' presentation was performed and they were classified as having a mild, moderate or severe presentation.

A mild presentation refers to patients without shortness of breath, dyspnea or abnormal chest imaging. A moderate presentation refers to patients who have evidence of lower respiratory disease with oxygen saturation $>94 \%$ on room air. Patients classified as having a severe presentation have a respiratory rate more than 30 breaths per minute, an oxygen saturation $<94 \%$ on room air, partial arterial pressure of oxygen to inspired oxygen fraction ratio $\left(\mathrm{PaO}_{2} / \mathrm{FiO}_{2}\right)$ $<300$, and lung infiltrates occupying $>50 \%$ of the lung fields.

\section{Results}

A total of 14 cases were included in the study. The majority of the patients were males $(85.71 \%)$. The mean age was 54 years. Thirteen patients $(92.85 \%)$ had severe illness and only 1 patient had a moderate presentation. Almost two thirds of the patients died (64.28\%). Most

Table 1: Most common presenting symptoms of the patients in the study.

\begin{tabular}{|l|c|}
\hline \multicolumn{1}{|c|}{ Symptoms } & Percentage (count) \\
\hline Fever & $78.57 \%(11)$ \\
\hline Dyspnea & $85.71 \%(12)$ \\
\hline Dry cough & $92.85 \%(13)$ \\
\hline Myalgia/arthralgia & $42.84 \%(7)$ \\
\hline Sore throat & $7.15 \%(1)$ \\
\hline Headache & $14.3 \%(2)$ \\
\hline Loss of taste & $0 \%(0)$ \\
\hline Anosmia & $0 \%(0)$ \\
\hline Gastrointestinal symptoms & $21.45 \%(3)$ \\
\hline Chest pain & $7.15 \%(1)$ \\
\hline
\end{tabular}

Austin J Infect Dis - Volume 8 Issue 1 - 2021

Submit your Manuscript | www.austinpublishinggroup.com

Salloum et al. (C) All rights are reserved
Citation: Fakhreddine S, Jaber R, Skaff E, Salloum S and Maatouk A. Case Series of Pneumomediastinum in COVID-19 Infection. Austin J Infect Dis. 2021; 8(1): 1043. 

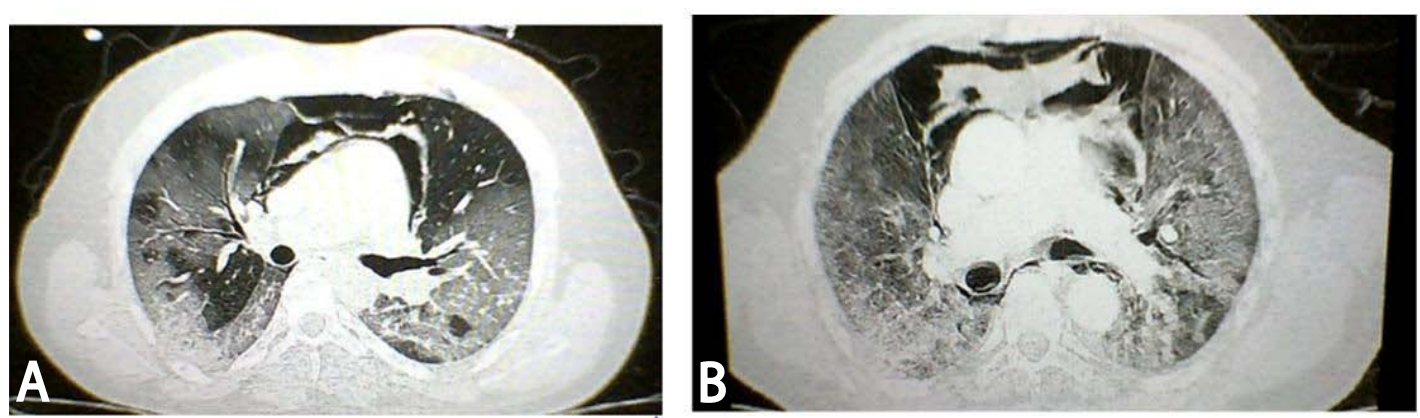

Figure 1: Chest CT scan from two patients with COVID-19 who developed pneumomediastinum. (A)A 33-year-old man who had severe pneumomediastinum (arrow) without positive pressure ventilation. (B) A65-year-old man, who was on NIPPV (noninvasive positive pressure ventilation), developed severe pneumomediastinum (arrows).

Table 2: Labs of the patients on admission.

\begin{tabular}{|c|c|c|c|c|c|c|c|c|c|c|c|c|c|c|c|}
\hline Lab & Patient 1 & Patient 2 & Patient 3 & Patient 4 & Patient 5 & Patient 6 & Patient 7 & Patient 8 & Patient 9 & Patient 10 & Patient 11 & Patient 12 & Patient 13 & Patient 14 & Mean \\
\hline WBCK ImicL & 15 & 24.87 & 20.95 & 6.16 & 18.54 & 25.2 & 2.5 & 29.87 & 28.55 & 28.79 & 16.37 & 15.87 & 23 & 30 & 24.28 \\
\hline Neut \% & 90 & 92.8 & 96.8 & 92.2 & 92.2 & 93.8 & 76 & 92.8 & 96.2 & 95.1 & 90 & 91 & 93 & 96 & 91.99 \\
\hline Hb gldl & 11.8 & 5.2 & 5.5 & 13.2 & 10.3 & 7.2 & 14.6 & 9.9 & 11.1 & 10.8 & 14 & 15.8 & 14 & 12 & 11.04 \\
\hline PTT Sec & N/A & 177 & 30 & 32 & 31 & 85 & 32 & 41 & 31 & 91 & 31 & 39 & 43 & 55 & 55.23 \\
\hline CRP mgldl & N/A & 18.6 & 37.7 & 17.6 & 21 & 30.4 & 11.5 & 21 & 28.2 & 29.5 & 10 & 5.9 & 14.1 & 21.8 & 20.56 \\
\hline INR & N/A & $>8$ & 1 & 1 & 1 & 1.58 & 1 & 1 & 1 & 6.19 & 1 & 1 & 2.48 & 1 & 1.6 \\
\hline PLT KImicL & 400 & 297 & 102 & 93 & 71 & 25 & 130 & 126 & 90 & 33 & 295 & 174 & 75 & 66 & 141.2 \\
\hline Crea mgldl & 1 & 0.6 & 2 & 0.9 & 1.3 & 3.2 & 0.9 & 5.3 & 2.7 & 4.2 & 1 & 1.4 & 3.5 & 1.6 & 2.18 \\
\hline AST UIL & $\mathrm{N} / \mathrm{A}$ & 754 & 63 & 46 & 66 & 39 & 397 & 206 & 42 & 39 & 85 & 49 & 574 & 72 & 187 \\
\hline ALT UIL & 47 & 519 & 68 & 63 & 33 & 30 & 381 & 316 & 19 & 34 & 185 & 34 & 382 & 49 & 154.2 \\
\hline CPK UIL & N/A & N/A & 66 & 228 & 165 & 199 & N/A & 596 & 145 & N/A & N/A & N/A & 840 & 651 & 361.2 \\
\hline Dd nglmL & 7840 & 14202 & 10000 & 1340.4 & 1981.8 & 4582 & 155 & 5628 & 5376 & 7756 & 257 & 482 & 10000 & 10000 & 5685.7 \\
\hline Fer nglmL & 416 & 705 & 1223 & 766.8 & N/A & 3286 & 10500 & 18564 & 2150 & 40000 & 11546 & 5599 & 2181 & 7000 & 7995.1 \\
\hline LDH UIL & N/A & 740 & 496 & 403 & N/A & 714 & 283 & 421 & 362 & 6534 & N/A & 438 & 1268 & N/A & 1165.9 \\
\hline PCt nglmL & N/A & 0.14 & 3.16 & 0.16 & 0.43 & 1.52 & 0.18 & 4.57 & 1.62 & 1.12 & 0.13 & 0.24 & 5.96 & 5.99 & 1.94 \\
\hline
\end{tabular}

Neut: neutrophils; Hb: hemoglobin; Crea: Creatinine; AST: Aspartate Transaminase; ALT: Alanine Transaminase; Dd: D-dimer; Fer: Ferritin; PLT: Platelet; PCT: Proalcitonine; CPK: Creatinine Phosphokinase; Trop: Troponin; LDH: Lactate Dehydrogenase.

commonly, the patients presented with a dry cough $(92.85 \%)$, followed by dyspnea, fever, myalgia, gastrointestinal symptoms, headache, decrease oral intake (Table 1).

The average time till death was 8.8 days. Around $35.72 \%$ of the patients were discharged home with $40 \%$ requiring oxygen therapy at home. The average hospital stay was 15.21 days. One patient was transferred from another hospital. Around $64.3 \%$ of the patients needed admission to an Intensive Care Unit (ICU) mainly due to severe respiratory failure and the need for invasive or noninvasive mechanical ventilation. One of the patients needed ICU admission because of atrial fibrillation with hemodynamic instability. Five patients $(35.72 \%)$ developed pneumomediastinum without any kind of mechanical ventilation during hospitalization. In addition, the majority of patients who were treated with invasive mechanical ventilation had pneumomediastinum before intubation but were on noninvasive positive pressure ventilation. Only 2 patients developed this complication after invasive mechanical ventilation on days 1 and
5 (Figure 1).

We have found that upon admission most of the patients showed at least one elevated inflammatory marker such as White Blood Cells (WBC), neutrophil percentage, C-Reactive Protein (CRP), D-dimer, ferritin and procalcitonin. The results are shown in (Table 2).

Comparison of the means of WBC, neutrophils percentage, CRP, creatinine, ALT, AST, procalcitonin, ferritin and D-dimer levels was done between the group of patient who died and those who survived. These numbers were higher in the patients who died. In addition, hemoglobin levels and platelets means were lower in the patients who died. The results are shown in (Table 3).

\section{Discussion}

Our results showed evidence supporting the presence of pneumomediastinum in COVID-19 patients without even any use of positive pressure mechanical ventilation. Many reports described cases of pneumomediastinum that occurred without any positive 
Table 3: Mean comparison of lab results of patients who died and those who survived.

\begin{tabular}{|c|c|c|c|c|c|c|c|c|c|c|c|}
\hline & WBC & Neut & $\mathrm{Hb}$ & PLT & CRP & Crea & ALT & AST & Proc & Fer & Dd \\
\hline Dead & 25.23 & 94.3 & 9.5 & 98.3 & 24.7 & 2.82 & 161.2 & 206 & 2.73 & 9388.6 & 7725 \\
\hline Survivor & 11.3 & 87.8 & 13.7 & 218.4 & 11.25 & 1.04 & 142 & 144.5 & 0.17 & 5765.5 & 2014 \\
\hline
\end{tabular}

airway pressure mechanical ventilation $[6,7]$. This can point to a direct pathophysiological effect resulting from the virus itself. One study showed that the occurrence of pneumomediastinum was higher in COVID-19 ARDS than in non-COVID-19 ARDS when using the same ventilatory protective settings [2]. Some proposed that an alveolar damage caused by the infection added to alveolar wall rupture from strenuous coughing is the cause of pneumomediastinum [8].

Most of our patients were males. This is in accordance to what is described in the literature where most of the patients who develop spontaneous pneumomediastinum were males [9].

The most common clinical manifestation of COVID-19 infection was dry cough. Furthermore, most of the patients who developed a pneumomediastinum had severe presentation. Physical findings of pneumomediastinum may be hard to detect. However, the most remarkable one is subcutaneous emphysema, other signs detectable are fever, Hamman's sign which is an auscultative sign referring to crackles generated by the heart beats [10]. Tachycardia, tachypnea and hypotension can also occur [7].

Patients who died, had more disturbed laboratory findings in comparison with those who survived.

The average time till death was 8.8 days and the average hospital stay was 15.21 days. This is close to what was seen in a study that showed that the length of stay in the hospital was 18 days. Concerning the in-hospital mortality, it was $56.5 \%$, slightly less than what we observed in our case series (64.28\%) [2]. This could be due to low number of patients in both studies (14 patients in our study and 23 patients in the other one). It is generally considered that, by itself, pneumomediastinum is a benign disease [9]. However, it has been suggested that the pneumomediastinum in COVID-19 can be a predictor of poor outcome [11]. This can be due to the fact that a pneumomediastinum is more likely to occur in patients with severe disease. In all cases, confirmation requires larger studies comparing patients with pneumomediastinum with similar patients without this complication.

\section{Conclusion}

Pneumomediastinum can occur with COVID-19 infections. It affects males more than females. Most patients have a severe disease at presentation. The mortality rates were high in patients with pneumomediastinum reaching $64.28 \%$.

\section{Ethical Approved}

No ethical considerations were violated, collection of the data was made anonymously. No patient identifiers collected. Approval for data collection was taken from the hospital.

\section{References}

1. Mohan V, Tauseen RA. Spontaneous pneumomediastinum in COVID-19. BMJ Case Rep. 2020; 13: e236519.

2. Lemmers $\mathrm{DHL}$, Abu Hilal $\mathrm{M}$, Bnà $\mathrm{C}$, Prezioso $\mathrm{C}$, Cavallo $\mathrm{E}$, Nencini $\mathrm{N}$, et al. Pneumomediastinum and subcutaneous emphysema in COVID-19: barotrauma or lung frailty? ERJ Open Res. 2020; 6: 00385-2020.

3. Kouritas VK, Papagiannopoulos K, Lazaridis G, Baka S, Mpoukovinas I Karavasilis V, et al. Pneumomediastinum. Journal of Thoracic Disease. 2015; 7.

4. Slutsky AS, Ranieri VM. Ventilator-induced lung injury. N Engl J Med. 2013 369: 2126-2136.

5. Ioannidis G, Lazaridis G, Baka S, Mpoukovinas I, Karavasilis V, Lampaki S, et al. Barotrauma and pneumothorax. J Thorac Dis. 2015; 7: S38-S43.

6. Quincho-Lopez A, Quincho-Lopez DL, Hurtado-Medina FD. Case Report: Pneumothorax and Pneumomediastinum as Uncommon Complications of COVID-19 Pneumonia-Literature Review. Am J Trop Med Hyg. 2020; 103: 1170-1176.

7. Goldman N, Ketheeswaran B, Wilson H. COVID-19-associated pneumomediastinum. Clin Med (Lond). 2020; 20: e91-e92.

8. López Vega JM, Parra Gordo ML, Diez Tascón A, Ossaba Vélez S. Pneumomediastinum and spontaneous pneumothorax as an extrapulmonary complication of COVID-19 disease. Emerg Radiol. 2020; 27: 727-730.

9. Shan S, Guangming L, Wei L, Xuedong Y. Spontaneous pneumomediastinum, pneumothorax and subcutaneous emphysema in COVID-19: case report and literature review. Rev Inst Med Trop Sao Paulo. 2020; 62: e76.

10. Park SJ, Park JY, Jung J, Park SY. Clinical Manifestations of Spontaneous Pneumomediastinum. Korean J Thorac Cardiovasc Surg. 2016; 49: 287-291.

11. Al-Azzawi M, Douedi S, Alshami A, Al-Saoudi G, Mikhail J. Spontaneous Subcutaneous Emphysema and Pneumomediastinum in COVID-19 Patients: An Indicator of Poor Prognosis? Am J Case Rep. 2020; 21: e925557. 